\title{
Síndrome ascítica em frangos de corte: uma revisão sobre a fisiologia, avaliação e perspectivas
}

\author{
Ascitic syndrome in broiler chickens: a review about physiology, evaluation and \\ perspectives
}

\author{
Millor Fernandes do Rosário ${ }^{1}$ Marco Aurélio Neves da Silva ${ }^{2}$ \\ Antonio Augusto Domingos Coelho ${ }^{3}$ Vicente José Maria Savino ${ }^{3}$
}

\section{- REVISÃO BIBLIOGRÁFICA -}

\section{RESUMO}

Os programas de melhoramento genético de frangos de corte que buscam máxima velocidade de ganho de peso, alta eficiência alimentar, alta viabilidade, maior rendimento de carcaça e menor deposição de gordura podem desencadear algumas síndromes fisiológicas, dentre as quais destacam-se o estresse calórico, a morte súbita e a ascite. A ascite se enquadra no conceito das síndromes multifatoriais, uma vez que sua manifestação ocorre quando certos fatores genéticos e ambientais atuam em conjunto determinando o processo. As limitações anatômica e fisiológica da circulação sanguínea nos pulmões provocam a síndrome de hipertensão pulmonar (PHS); esta pode provocar grande acúmulo de fluido na cavidade abdominal, quadro este denominado de ascite. Ocorre redução da eficiência da circulação sangüínea, levando as aves à morte por hipóxia, predominantemente no período entre 30 e 40 dias de idade. Uma vez desencadeado o processo ascítico, a ave dificilmente é aproveitada no abate já que a mesma restringe o consumo de alimento, ganhando menos peso. Adicionalmente, a carcaça apresenta aumento do volume da cavidade abdominal e conseqüente congestão dos órgãos internos. A descrição da fisiologia, medidas de avaliação e perspectivas são apresentadas neste trabalho.

Palavras-chave: ambiente, ascite, coração, frango de corte, genética, pulmão.

\section{ABSTRACT}

Poultry genetic breeding programs which look for maximum weight gain, improved feed conversion, high viability, high carcass yield and low fat deposition may cause some physiologic syndromes, for example caloric stress, sudden death and ascites. Ascites is framed in concept of the multifactorial syndromes, once its manifestation happens when certain genetic and environment factors act together, determining the process. Anatomical and physiological limitations in blood circulation in the lungs give rise to pulmonary hypertension syndrome (PHS), producing great fluid accumulation in abdominal cavity, which is denominated ascites. Ascites causes reduction of blood circulation efficiency, leading broilers to death due to hypoxia, mainly between 30 and 40 days of age. Ascitic broilers have reduced body weight gain as a consequence of feed restriction. Additionally, there is increased abdominal cavity volume and internal organ congestion. Ascites is discussed through this manuscript based on physiological description, evaluation measures and perspectives.

Key words: environment, ascites, heart, broiler chicken, genetics, lung.

\section{INTRODUÇÃO}

A produção mundial de carne de frangos ultrapassou 49 milhões de toneladas no ano de 2002, destacando-se os Estados Unidos, o Brasil e a China com $14,5,7,3$ e 5,4 milhões de toneladas, respectivamente, tendo o Brasil crescido $12 \%$ na produção em relação ao ano de 2001 (AVEWORLD, 2003). A evolução da avicultura brasileira deu-se principalmente a partir da década de $60 \mathrm{com}$ a introdução de materiais genéticos importados, especializados em produção de carne e ovos. Além do melhoramento genético, as áreas de sanidade,

${ }^{1}$ Engenheiro Agrônomo, Mestre em Agronomia, Departamento de Genética, Escola Superior de Agricultura “Luiz de Queiroz”, Universidade de São Paulo (ESALQ/USP). Av Pádua Dias, 11, CP 83, Vila Independência, 13400-970, Piracicaba, SP, Brasil. Email: mfrosari@esalq.usp.br. Autor para correspondência.

${ }^{2}$ Engenheiro Agrônomo, Mestre em Agronomia, Bolsista de Doutorado da CAPES, Departamento de Genética, Escola Superior de Agricultura "Luiz de Queiroz", USP.

${ }^{3}$ Professor Doutor, Departamento de Genética, ESALQ/USP. 
nutrição, ambiência e manejo contribuíram significativamente para que resultados positivos fossem alcançados.

Um frango de corte que levava 120 dias para atingir 1.500g em 1920, em 1980 levava 44 dias e, em 1998, apenas 33 dias para atingir o mesmo peso (ALBERS, 1998). Com o passar dos anos, a demanda provocada pela exigência do consumidor e transferida para toda a cadeia de produção avícola, evoluiu para animais com menor teor de gordura e mais carne, exigindo das empresas e dos criadores maiores cuidados na seleção dos reprodutores e na criação dos frangos de corte (SAUVEUR, 1991; REFFAY, 1998). Dessa forma, os programas de melhoramento genético, na busca por máxima velocidade de ganho de peso, alta eficiência alimentar, alta viabilidade, maior rendimento de carcaça e menor deposição de gordura desencadearam também síndromes fisiológicas, dentre as quais se destacam o estresse calórico, a morte súbita e a ascite (SANCHEZ et al., 2000).

Informações provenientes de 18 países de quatro continentes identificaram $4,7 \%$ de aves descartadas dos plantéis por incidência de ascite, com perdas que totalizaram aproximadamente um bilhão de dólares no ano de 1996, sendo que, no Brasil, a incidência foi de 0,4 a 0,6\% e nos Estados Unidos e Reino Unido foi de 1,4\% da produção nacional de cada país (MAXWELL \& ROBERTSON, 1997).

GONZALES \& MACARI (2000)

mostraram dados do estado de São Paulo, de 1998, segundo os quais 337.633.015 aves abatidas sob inspeção federal, 227.308 foram condenadas por apresentarem ascite (acúmulo de líquido na cavidade abdominal). A ascite foi a terceira causa de condenação no abatedouro, correspondendo a 14,6\% das aves condenadas depois de caquexia $(34,6 \%)$ e aspecto repugnante $(18,3 \%)$.

Devido à importância do presente assunto, nesta revisão, serão abordadas a descrição da fisiologia, medidas de avaliação e perspectivas sobre a síndrome ascítica em frangos de corte.

\section{A FISIOLOGIA}

Os frangos de corte vêm sendo selecionados em programas de melhoramento para elevado peso vivo, o que vem resultando em um substancial aumento na velocidade de crescimento. As limitações anatômicas e fisiológicas da circulação sanguínea nos pulmões levam a ave a uma síndrome de hipertensão pulmonar (PHS), que pode levar a um grande acúmulo de fluido na cavidade abdominal, quadro este denominado de ascite (HUCHZERMEYER \& DERUYCK, 1986; JULIAN, 1993). Ocorre uma redução da eficiência da circulação sangüínea, levando à morte das aves por hipóxia, no período entre 30 a 40 dias de idade, conforme DALE (1990). Esse quadro foi detectado décadas atrás em regiões de altas altitudes, mas atualmente também é encontrado em locais de baixa altitude, onde os frangos, por possuírem restrições anatômicas e fisiológicas do sistema cardio-respiratório, encontram-se com baixa oxigenação durante os períodos de rápido crescimento (HERNANDES, 2000).

$\mathrm{O}$ processo que mais freqüentemente leva os frangos à ascite foi muito bem descrito por JULIAN (1987) e GONZALES \& MACARI (2000). Devido a uma insuficiente oxigenação, o ritmo cardíaco aumenta na tentativa de suprir mais oxigênio para o metabolismo oxidativo dos tecidos em rápido crescimento, causando uma hipertensão pulmonar. Com uma prolongada falta de oxigênio, mecanismos de regulação do organismo da ave são acionados para manter a homeostase. Em resposta a tal estímulo, a medula passa a produzir maior número de hemácias, o que agrava a hipertensão pulmonar e altera os parâmetros hematológicos (MACARI et al., 1994).

O quadro é agravado ainda mais pelo aumento da resistência ao fluxo sangüíneo no pulmão, desequilíbrio entre a necessidade e o fornecimento de oxigênio e insuficiência cardíaca direita. A predisposição à ascite é maior nos frangos porque o pulmão é rígido e fixo na cavidade torácica e o peso do órgão em relação ao peso corporal diminui em função da idade (GONZALES \& MACARI, 2000).

$O$ déficit de oxigênio causa aumento na concentração de hemoglobina, no hematócrito e no número de eritrócitos, com conseqüente aumento da viscosidade sangüínea. À medida que aumenta a pressão arterial pulmonar, ocorre a hipertrofia cardíaca do lado direito.

A hipertensão pulmonar crônica resulta na hipertrofia do ventrículo direito, e as aves suscetíveis apresentam valor da relação ventrículo direito / ventrículo total acima de 0,37 (LUBRITZ \& McPHERSON, 1994), além de causar mau funcionamento da válvula átrio ventricular direita, que acaba permitindo refluxo do sangue venoso dentro da veia cava. Isso leva à congestão do fígado e ao extravasamento de líquido pela sua superfície. Quando a taxa de extravasamento é maior que a capacidade das membranas abdominais em absorver o líquido, a ascite se desenvolve. Isso eventualmente conduz à morte por falha respiratória causada pela pressão do líquido nos sacos aéreos (JULIAN et al., 1989). 
As aves com ascite apresentam um quadro clínico mórbido caracterizado por anorexia, perda de peso, respiração ofegante e imobilidade. As canelas se tornam progressivamente desidratadas, sem brilho e a crista e barbelas têm uma coloração cianótica. As penas ficam arrepiadas e a ave permanece deprimida, não se alimenta ou bebe água. Nos quadros mais avançados de ascite, o abdômen fica dilatado, percebendo-se à palpação a presença de líquido na cavidade abdominal. Nessas condições, a simples manipulação da ave para um exame clínico pode resultar em sua morte (GONZALES \& MACARI, 2000).

Quando a síndrome ascítica se instala, os níveis hormonais de $\mathrm{T}_{3}$ e $\mathrm{T}_{4}$ se alteram como estudaram GONZALES et al. (1999) e DECUYPERE et al. (2000) os quais verificaram que os frangos de corte melhorados para maior taxa de crescimento podem apresentar alteração do metabolismo tiroidiano, em relação às aves não selecionadas. O hipotiroidismo funcional, sugerido por níveis plasmáticos de $\mathrm{T}_{3}$ (triiodotironina) e $\mathrm{T}_{4}$ (tiroxina) alterados, poderia determinar uma redução do consumo de oxigênio tecidual e, portanto, predispor as aves à hipoxemia, hipertensão pulmonar, hipertrofia ventricular direita e exsudação de fluido ascítico.

GONZALES \& MACARI (2000) comentaram que, apesar de a evolução do processo ascítico estar hoje bem esclarecida, o mecanismo bioquímico, iniciador de todo o processo, ainda é motivo de investigação científica. O estresse oxidativo celular pode estar envolvido, ou porque a célula tem uma proteção inadequada aos agentes oxidantes ou porque o alto metabolismo do frango gera uma quantidade grande de radicais livres, os quais, nas mitocôndrias, estão sendo apontados como os maiores responsáveis pelo processo de envelhecimento celular, além de estarem associados com doenças metabólicas. Estudos para elucidar o mecanismo bioquímico da ascite têm sido realizados por OLKOWSKI et al. (2001) e TANKSON et al. (2002a;b).

Uma vez desencadeado o processo ascítico, a ave restringe o consumo de alimento refletindo negativamente nos índices de desempenho zootécnico (ganho de peso e conversão alimentar) e se a ave não morrer até o final do período de criação comercial, sua carcaça é condenada no abatedouro.

Geralmente, o pico da ascite aparece entre a terceira e a quinta semana de idade das aves e os machos são mais predispostos a apresentar ascite do que as fêmeas, pois possuem maior crescimento corporal e conseqüentemente o metabolismo oxidativo dos machos é maior do que ao das fêmeas (MAXWELL et al., 1986).
Vale ressaltar que a ascite não é uma patologia, ou seja, não é causada por bactéria, vírus ou qualquer outro patógeno e do ponto de vista sanitário, a carcaça, apesar de apresentar uma aparência indesejável, pode ser consumida sem prejuízo à saúde humana. Porém, os abatedouros acabam por condenar as carcaças das aves ascíticas na linha de abate, mantendo padrões de qualidade da matéria-prima.

O mecanismo desencadeado pela síndrome ascítica em frangos de corte é ilustrado pela figura 1 .

\section{A AVALIAÇÃO}

A monitoria da síndrome ascítica não é realizada de forma regular pelos produtores de frangos, pois uma vez iniciado o processo, a evolução da doença é irreversível. Entretanto, em pesquisas e em programas de seleção para aumento de resistência genética à ascite, o monitoramento tem-se mostrado muito útil (GONZALES \& MACARI, 2000) e como esta síndrome está ligada diretamente ao sistema cardio-respiratório, parâmetros sangüíneos são utilizados indiretamente na avaliação da suscetibilidade ou resistência das aves à ascite.

Uma vez que a deficiência no suprimento de oxigênio para os tecidos em rápido crescimento é detectada pelos mecanismos homeostáticos da ave, maior número de hemácias são produzidas e os parâmetros hematológicos são alterados. Portanto, é possível monitorar a ascite em plantéis de frangos de corte, através de parâmetros fisiológicos como o valor hematócrito (SHLOSBERG et al., 1992; PRICE et al., 1998; ROSÁRIO et al., 2000 / 2002; SCHEELE et al., 2003a;b), a viscosidade sangüínea (MAXWELL et al., 1992; FEDDE \& WIDEMAN, 1996), o número de hemácias (STURKIE \& GRIMINGER, 1976), a relação ventrículo direito / ventrículo total (LUBRITZ \& McPHERSON, 1994), a porcentagem do fígado, baço e pulmão em relação ao peso corporal (SILVERSIDES et al., 1997), a temperatura corpórea (HERNANDES, 2000), o teor de hemoglobina (FEDDE et al., 1998), estudos bioquímicos (TANKSON et al.,2002a;b) e a análise de imagens digitais do coração (McGOVERN et al.,1999a; McGOVERN et al.,2000).

O valor hematócrito, o qual mensura a porcentagem de células sólidas sangüíneas, está diretamente ligado ao número de elementos figurados sangüíneos. Dentre eles, citam-se hemácias, basófilos, eosinófilos, heterófilos, leucócitos, monócitos e plaquetas. MAXWELL et al. (1992) relataram que frangos de corte ascíticos e não ascitícos apresentaram 


\begin{tabular}{|c|c|c|c|c|}
\hline $\begin{array}{l}\text { Ração de alta } \\
\text { energia, } \\
\text { peletizada, alto } \\
\text { nível de } \mathrm{Na}^{+}\end{array}$ & $\begin{array}{l}\text { Alta taxa de } \\
\text { crescimento }\end{array}$ & $\begin{array}{l}\text { Grandes } \\
\text { altitudes }\end{array}$ & \multirow[t]{2}{*}{$\begin{array}{l}\text { Variações } \\
\text { climáticas }\end{array}$} & $\begin{array}{l}\text { Patologias } \\
\text { pulmonares }\end{array}$ \\
\hline \multicolumn{4}{|c|}{$\Downarrow$} & \\
\hline \multicolumn{5}{|c|}{ Aumento da demanda de $\mathrm{O}_{2}$ (metabolismo oxidativo) } \\
\hline \multicolumn{5}{|c|}{$\Downarrow$} \\
\hline \multicolumn{5}{|c|}{ Mecanismos compensatórios: $\uparrow$ fluxo cardíaco, $\uparrow$ volume sistólico, $\uparrow$ retorno venoso } \\
\hline \multicolumn{5}{|c|}{$\Downarrow$} \\
\hline \multicolumn{5}{|c|}{$\uparrow$ débito cardíaco $=\uparrow$ fluxo sanguíneo } \\
\hline \multicolumn{5}{|c|}{$\Downarrow$} \\
\hline \multicolumn{5}{|c|}{ Hipertensão pulmonar } \\
\hline \multicolumn{5}{|c|}{$\Downarrow$} \\
\hline \multicolumn{5}{|c|}{$\begin{array}{l}\text { Dano pulmonar e bloqueio do trânsito sanguíneo } \\
\text { ( } \uparrow \text { pressão no pulmão e na artéria pulmonar) }\end{array}$} \\
\hline \multicolumn{5}{|c|}{$\Downarrow$} \\
\hline \multirow{3}{*}{\multicolumn{5}{|c|}{$\begin{array}{r}\uparrow \text { pressão no ventrículo direi } \\
\text { (espessamento da musculatura ventricular direita, insufi }\end{array}$}} \\
\hline & & & & \\
\hline & & & & $\uparrow$ pressão no átrio direito \\
\hline \multicolumn{5}{|c|}{$\Downarrow$} \\
\hline \multicolumn{5}{|c|}{$\begin{array}{l}\text { Refluxo de sangue do coração para o organismo (veias cavas), redução do retorno venoso, estase do } \\
\text { sistema porta-hepático (congestão crônica) }\end{array}$} \\
\hline \multicolumn{5}{|c|}{$\Downarrow$} \\
\hline \multicolumn{5}{|c|}{ Perda de líquido vascular: ASCITE } \\
\hline \multicolumn{5}{|c|}{$\Downarrow$} \\
\hline $\begin{array}{l}\uparrow \text { viscosidade do } \\
\text { sangue }\end{array}$ & 个 hematócrito & $\begin{array}{c}\uparrow \text { eritrócitos / } \\
\mathrm{mm}^{3}\end{array}$ & $\uparrow$ hemoglobina & $\begin{array}{c}\text { Hipertrofia do átrio e } \\
\text { ventrículo direito, flacidez } \\
\text { do músculo cardíaco }\end{array}$ \\
\hline
\end{tabular}

Figura 1 - Mecanismo desencadeado pela síndrome ascítica em frangos de corte, adaptado de GONZALES \& MACARI (2000).

valor hematócrito de $37,02 \% \pm 10,6$ e $27,06 \% \pm 2,6$, respectivamente. CUEVA et al. (1974) observaram um aumento considerável de glóbulos vermelhos, volume corpuscular médio, teor de hemoglobina nos glóbulos vermelhos e valores de hematócrito em frangos que apresentaram ascite criados em altitudes acima de 2.000 metros.

Para FURLAN et al. (1999), o hematócrito não deve ser analisado individualmente, pois pode ser influenciado pelo tamanho e pelo número de eritrócitos. Muitas pesquisas que abordam esse tipo de enfoque não mostraram essas relações, exceto em trabalhos que investigaram a síndrome de hipertensão pulmonar (OWEN et al., 1990; MIRSALINI \& JULIAN, 1991). Com o aumento da idade do frango de corte, foi observado que, apesar de o número de eritrócitos ter aumentado, o volume corpuscular médio diminuiu, não ocorrendo alteração significativa no valor do hematócrito. Essa proposta corrobora os achados de MAXWELL et al. (1990), que verificaram redução do hematócrito, mas um aumento do número de eritrócitos em aves submetidas à restrição alimentar.

Segundo MAXWELL et al. (1992), a viscosidade total do sangue determinada através do viscosímetro de Ostwald é um parâmetro que também pode ser utilizado para monitoramento da síndrome ascítica com valores de 2,141 $\pm 0,245$ centipoieses para aves normais e de 4,046 $\pm 1,034$ centipoieses para aves ascíticas, o que reflete acúmulo de excretas e solutos no sangue, devido à dificuldade de circulação do sangue e manutenção das condições ótimas para oxigenação das células.

WIDEMAN Jr. (1998) mostrou que a saturação de hemoglobina com oxigênio, em porcentagem, entre três incubações de frangos

Ciência Rural, v.34, n.6, nov-dez, 2004. 
suscetíveis à ascite e outras doenças, foi de 63,2\% e de $75,9 \%$ para machos ascíticos e não ascíticos, respectivamente e de $62,5 \%$ e de $74,2 \%$ para fêmeas ascíticas e não ascíticas, respectivamente.

Porém, estes parâmetros hematológicos empregados para monitorar a síndrome ascítica em frangos de corte, por serem indiretos e invasivos, isoladamente não fornecem informações precisas sobre a ascite (MAXWELL et al., 1992; SHLOSBERG et al., 1992; SILVERSIDES et al., 1997). Para sanar esta deficiência, emprega-se a metodologia direta de monitoramento desta síndrome, a qual necessita que a ave seja abatida. Esta técnica baseia-se na análise da relação ventrículo direito / ventrículo total (LUBRITZ et al., 1994; McGOVERN et al., 1999a). De acordo com LUBRITZ et al. (1995), aves que apresentaram valor dessa relação maior ou igual a 0,37 foram consideradas ascíticas, mas para JULIAN et al. (1992), quando esta relação estiver entre 0,25 a 0,299 deve-se suspeitar de uma moderada hipertrofia, podendo-se considerá-la severa quando acima de 0,299, ou normal, quando abaixo de 0,25.

A primeira reação do corpo à deficiência de oxigênio é o aumento da proporção do coração, a seguir ocorre alteração do número de hemácias e, em uma exposição continuada em condições de anóxia, uma série de eventos conduz aos sintomas clínicos de ascite. O aumento da proporção do coração e da viscosidade sangüínea conduzem ao aumento da pressão nos pulmões e à congestão pulmonar. Ocorre uma hipertorfia do ventrículo direito e o fígado tornase sobrecarregado. Os pesos do baço e do fígado e a densidade dos pulmões são um indicador geral desse estresse e que podem também ser utilizados para mensurar esta resposta fisiológica à deficiência de oxigênio (SILVERSIDES et al., 1997). Os dados destes mesmos autores, que conduziram um experimento envolvendo nove linhagens de frangos de corte, analisando o desempenho zootécnico e o desenvolvimento de órgãos (pulmão, fígado, baço e coração), sugerem que existem diferenças entre as linhagens avaliadas com relação à suscetibilidade ascítica.

Como a síndrome ascítica tem seu início de desenvolvimento entre 7 e 21 dias de idade, seria mais adequado que as análises hematológicas e anatômicas fossem realizadas neste período, estendendo-se até aos 42-49 dias de idade, a fim de correlacionar a incidência de ascite com as alterações hematológicas e anatômicas constatadas.

Portanto, verifica-se a necessidade de se combinar parâmetros hematológicos e anatômicos na tentativa de aumentar a precisão na avaliação da suscetibilidade ou resistência dos frangos de corte à ascite.

\section{GENÉTICA E AMBIENTE}

A ascite ou síndrome ascítica se encaixa no conceito de patologias avícolas consideradas multifatoriais, uma vez que é uma manifestação fisiológica que ocorre quando certos fatores genéticos, nutricionais, ambientais e de manejo atuam em conjunto. Isso exige estudos amplos, sendo de fundamental importância a inter-relação entre as variações ambientais e a resposta fisiológica da ave (JAENISCH et al., 1995). Vários fatores incluindo altitude, concentração de amônia, baixas temperaturas, crescimento rápido, dietas densas, reações vacinais, doenças respiratórias e suscetibilidade genética atuam produzindo deficiência de oxigênio nos tecidos (JULIAN, 1990).

Baixas temperaturas podem aumentar a hipertensão pulmonar e aumentar a atividade tireoideana. Nesse contexto, a ascite parece ter se tornado freqüente durante épocas frias (HERNANDEZ, 1987).

SCHEELE et al. (1992), trabalhando com duas linhagens de frangos de corte, obtidas pela seleção divergente para crescimento utilizando o frio como fator estressante, obtiveram maior valor hematócrito e maior mortalidade pela ascite nos frangos com rápido crescimento. Também SHLOSBERG et al. (1998), trabalhando com frangos de corte de três grupos genéticos obtidos de parentais selecionados pelo hematócrito (Alto, Médio e Baixo) e utilizando o frio como fator estressante, verificaram que entre as idades de 19 a 43 dias, as aves do grupo Baixo apresentaram menor valor hematócrito (35,50\%), atingiram maior peso corporal (1.802 g) e menor mortalidade por ascite (7\%) quando comparadas com as do grupo Alto, no qual a média para valor hematócrito foi de $41,55 \%$, o peso foi de 1.623 g e a mortalidade foi de $9 \%$.

WIDEMAN et al. (1998), utilizando uma dieta de 3.059 kcal EM. kg-1 e frio como fatores estressantes em frangos de corte de crescimento rápido, obtiveram médias significativamente diferentes de valor hematócrito para machos ascíticos (40,3\%) e não ascíticos (35,8\%) e para fêmeas ascíticas (41,3\%) e não ascíticas (36,8\%).

A utilização da restrição alimentar no estudo da ascite foi empregada por SCHEELE \& FRANKENHUIS (1988) mostrando que o aumento do conteúdo em energia metabolizável do alimento (de 3.273 a 3.631 kcal EM.kg-1) leva a um aumento 
significativo da taxa do hematócrito nos frangos de crescimento rápido, mas não nos de crescimento lento. Quando duas linhagens de frangos de corte obtidas pela seleção divergente para crescimento (rápido e lento) foram comparadas, as taxas de hematócrito e de mortalidade por ascite foram significativamente mais elevadas para as aves de rápido crescimento do que para as crescimento lento (SCHEELE et al., 1992).

McGOVERN et al. (1999b) avaliaram o efeito da restrição alimentar (ad libitum e 18g de alimento/ave/dia dos 7 aos 15 dias de idade) e a aplicação de óleo de canola sobre a palha onde os frangos foram alojados, a fim de conter a poeira sobre performance de crescimento, conformação de carcaça e incidência de ascite em 800 machos e concluíram que a restrição alimentar foi capaz de reduzir a mortalidade por ascite de $15,9 \%$ para $6,3 \%$ e a aplicação de óleo de canola na cama melhorou significativamente a qualidade do ar dentro do aviário e também melhorou a morfologia do coração, avaliada através da análise digital de imagens do coração, reduzindo a área do ventrículo direito de 0,44 para $0,36 \mathrm{~cm}^{2}$.

Outros trabalhos que empregaram a restrição alimentar para reduzir a incidência de mortalidade por ascite foram realizados por PEACOCK et al. (1989), REEVES et al. (1991), SHLOSBERG et al. (1991), ACAR et al. (1995), LOTT et al. (1996) e BUYS et al. (1998).

A restrição alimentar qualitativa pode ser obtida manipulando-se o conteúdo de energia da dieta, relações entre constituintes da dieta (proteína, energia, minerais, vitaminas) e forma de apresentação (farelada ou peletizada) e deve ser levada em conta nas primeiras três semanas de vida da ave, a fim de se reduzir a incidência de ascite (ALBERS et al., 1990; SHLOSBERG et al., 1991; SCHEELE, 1993).

McGOVERN et al. (2000) também estudaram o efeito da flutuação da temperatura (durante o dia - das 6 às 18 horas de mais $3^{\circ} \mathrm{C}$ e durante a noite - das 18 às 6 horas de menos $3^{\circ} \mathrm{C}$ ) e da aplicação de óleo de canola sobre a palha onde os frangos foram alojados e concluíram que a flutuação na temperatura resultou em um aumento de 1,4\% na mortalidade por ascite em relação ao tratamento controle (sem flutuação e sem aplicação de óleo de canola) e que os frangos do tratamento controle apresentaram o menor peso do ventrículo direito em relação aos frangos do tratamento com flutuação de temperatura.

FEDDE et al. (1998) mostraram que aves em estado de hipoxemia apresentaram maior teor de hemoglobina no sangue após três horas de restrição alimentar, porém isto não foi causado nem pela diminuição do consumo de oxigênio nem pela limitação da captação de oxigênio pelo bico da ave. Os dados destes autores também demonstraram que a restrição alimentar resultou em um controle do fluxo aerodinâmico, o qual poderia diminuir a vasoconstrição pulmonar e as falhas no ventrículo direito, reduzindo, assim, a mortalidade por ascite.

As trocas de ar inadequadas aumentam as concentrações de partículas de monóxido de carbono (CO), dióxido de carbono $\left(\mathrm{CO}_{2}\right)$ e amônia $\left(\mathrm{NH}_{3}\right)$ e diminuem as de oxigênio $\left(\mathrm{O}_{2}\right)$, favorecendo a ascite. BEKER et al. (2003) estudaram os efeitos da concentração de oxigênio (12,14,16,18 e 20,6\%) sobre a propensão ao desenvolvimento da síndrome ascítica em machos e concluíram que, com a diminuição da concentração de oxigênio no ambiente, a massa do ventrículo direito e valor hematócrito e outros parâmetros aumentaram significativamente, sendo que o hematócrito parece ser o mais sensível indicador da mudança fisiológica atribuída ao oxigênio atmosférico. Os dados obtidos também sugerem que uma concentração de oxigênio de 19,6\% é o nível mínimo para se evitar mudanças cardíacas e do valor hematócrito com relação ao desenvolvimento da ascite.

$\mathrm{O}$ aquecimento inicial dos pintinhos, principalmente nas regiões mais frias, como a região sul do Brasil, parece estar relacionado com o desenvolvimento da ascite. É interessante criar condições de renovação de ar no interior dos galpões, mas não em detrimento do aquecimento dos pintinhos na fase inicial de criação. A utilização de estufas nos pinteiros, quando a renovação do ar é adequada, proporciona maior conforto térmico, reduzindo a mortalidade por ascite (GONZALES \& MACARI, 2000).

JAENISCH et al. (1995) realizaram um experimento para avaliar o efeito da utilização de pinteiros (estufas) cobertos na redução da síndrome ascítica em frangos de corte e concluíram que as estufas proporcionaram maior conforto térmico aos frangos, sem comprometer os parâmetros hematológicos (hematócrito e hemoglobina), reduzindo a mortalidade por ascite e o consumo de gás dos aquecedores.

Na região sul do Brasil, é muito comum o uso de aquecedores à lenha; já na região sudeste do Brasil os aquecedores a gás são preferidos. Mas os dois tipos têm performance adequada para manter o conforto térmico dos pintinhos durante os primeiros dias de vida, reduzindo a ascite.

Relatos de maior incidência de ascite em frangos de corte têm sido constatados em altitudes acima de 2.000 metros em relação ao nível do mar, 
devido à rarefação do oxigênio atmosférico, o que leva à hipóxia tecidual e aumento do débito cardíaco (GONZALES \& MACARI, 2000).

$\mathrm{O}$ efeito das altas e baixas altitudes foi muito bem estudado nos trabalhos de CUEVA et al. (1974), JULIAN \& WILSON (1992), YERSIN et al. (1992), KANNAN \& SINGH (1994) e OWEN et al. (1995), sendo que nos dias atuais é possível encontrar registros de mortalidade por ascite tanto em altas quanto em baixas altitudes.

Vários fatores relacionados à sanidade do lote de frangos e às medidas no controle sanitário dos plantéis podem favorecer a incidência de ascite. Entre esses fatores, podem-se citar: fumigação excessiva com formol (nascedouros e galpões), complicações por aspergilose pulmonar e broncopneumonias, problemas tóxicos que afetam o fígado, coração ou pulmão, pintos de baixa qualidade resultantes de nascimentos retardados ou com problemas pulmonares e vacinação por pulverização, que pode causar danos ao sistema respiratório dos pintinhos de um dia de idade (GONZALES \& MACARI, 2000).

TANKSON et al. (2002b) relataram que Enterococcus faecalis, uma bactéria que vive no abdômen dos frangos de corte, causa ascite em frangos. O objetivo deste trabalho foi determinar se culturas congeladas ou autoclavadas de $\boldsymbol{E}$. faecalis reteriam a habilidade de provocar ascite e estes autores concluíram que tanto sob a forma congelada quanto autoclavada, as culturas desta bactéria foram capazes de causar ascite. Aparentemente, E. faecalis causa ascite em frangos pela produção e liberação de uma toxina desconhecida. Em outro trabalho com o mesmo patógeno, TANKSON et al. (2002a) relataram que recentes estudos têm mostrado que $\boldsymbol{E}$. faecalis causa ascite em $97 \%$ das aves dentro de 48 horas após a contaminação.

O excesso de sódio na dieta pode induzir ascite em frangos de corte (JULIAN, 1993), sendo que as aves próximas aos 21 dias de idade são muito sensíveis a este excesso (JULIAN et al., 1992) e a toxicidade por este mineral, nesta idade, é muito comum (JULIAN, 1996). Outra fonte de excesso de sódio é a água de bebida, que é mais prejudicial ainda, pois a ave consome 2,4 vezes mais água do que alimento por dia, dentro de um mesmo peso base (WILLIAMS, 1996). Alguns trabalhos têm mostrado os efeitos de vários níveis de sódio na água sobre a incidência de ascite (JULIAN, 1987; JULIAN et al., 1992), mas muitos experimentos sobre ascite têm ignorado esta possível contaminação da água pelo sódio, podendo tornar inválidas as conclusões obtidas.
Em uma condição de acidose sangüínea, a ave está mais propensa a desenvolver a ascite, pois ocorre uma vasoconstrição pulmonar, dificultando as trocas gasosas e a circulação sangüínea; já em uma condição de alcalose sangüínea, ocorre uma vasodiltação pulmonar inibindo ou diminuindo a mortalidade por ascite.

Várias fontes de sódio foram estudadas e foi concluído que o cloreto de amônio (BRANTON et al., 1986; SHLOSBERG et al., 1998) e o cloreto de sódio (SHLOSBERG et al., 1998) favoreceram a acidose e conseqüentemente, a ascite; já o bicarbonato de sódio (BOTTJE \& HARRISON, 1985) e o carbonato de sódio (OWEN et al., 1994) favoreceram a alcalose.

Por isso, recomenda-se que a ração e a água de bebida das aves sejam examinadas analiticamente para se conhecer o pH e a composição iônica das mesmas, evitando ou reduzindo, dessa forma, favorecer o desenvolvimento de ascite pelas aves.

Então, medidas em curto prazo como o emprego da restrição alimentar ou uma dieta com menor valor calórico nas primeiras semanas de idade da ave, aquecimento adequado, controle do teor de sódio na dieta e na água de bebida, controle da contaminação por micotoxinas são válidas para se reduzir a incidência de ascite em frangos de corte.

\section{PERSPECTIVAS}

Programas de melhoramento genético vêm sendo realizados em várias partes do mundo utilizando altas altitudes, câmaras hipobáricas e seleção para parâmetros sangüíneos. A herdabilidade para o índice cardíaco (relação do peso do ventrículo direito pelo ventrículo total) de $0,27 \pm 0,08$ e para a incidência de ascite de $0,44 \pm 0,09$, relatadas por LUBRITZ et al. (1995) sugerem que a seleção baseada nas médias das famílias reprodutoras para diminuição do índice cardíaco e mortalidade por ascite podem ser efetivas.

A seleção tende a eliminar os indivíduos suscetíveis da população, por isso, programas de melhoramento têm sido praticados em altas altitudes ou sob condições de frio, onde apenas os resistentes sobrevivem. Essa seleção provoca aumento da mortalidade nos plantéis de melhoramento, podendo reduzir a variabilidade genética para taxa de crescimento e conversão alimentar, e reduz a intensidade de seleção para características essenciais (ganho de peso, conversão alimentar, rendimento de carcaça). Procedimentos de seleção controlada, preservando ao máximo o potencial de seleção são 
por essa razão preferidos quando se pratica a seleção para resistência à ascite (WIDEMAN, 1988).

SANCHEZ et al. (2000) fizeram alguns relatos a respeito do uso da seleção de aves resistentes à síndrome ascítica visando diminuir as perdas da indústria avícola francesa. Os autores utilizaram critérios indiretos de seleção como o hematócrito, volume pulmonar, eletrocardiograma e a troponina $\mathrm{T}$ e critérios diretos como seleção em altas altitudes, onde o ar é mais rarefeito. Assim, as aves resistentes seriam selecionadas para constituir os parentais das progênies comerciais.

Atualmente as empresas optam por conviver com uma certa incidência de ascite a desenvolver um programa de melhoramento genético exclusivo para obter linhagens resistentes, pelo fato de que provavelmente, qualquer linhagem que apresente resistência à ascite será penalizada por uma menor performance zootécnica em relação as demais linhagens comercializadas.

\section{CONCLUSÃO}

A ascite é uma síndrome que provoca sérios prejuízos à indústria avícola e, portanto, deve ser monitorada de forma criteriosa, utilizando-se parâmetros hematológicos e anatômicos na seleção de aves resistentes. É necessário adotar medidas de manejo da nutrição, sanidade, aquecimento, ventilação para que as linhagens comerciais, atualmente criadas, não apresentem ou reduzam a mortalidade por ascite. Até o presente momento, a obtenção de materiais genéticos resistentes à ascite implica prejuízo ao desempenho zootécnico, fato este que vem dificultando as empresas desenvolverem programas de melhoramento genético específicos para ascite.

\section{REFERÊNCIAS BIBLOGRÁFICAS}

ACAR, N. et al. Growth of broiler chickens in response to feed restriction regimens to reduce ascites. Poultry Science, v.74, n.5, p.833-843, 1995.

ALBERS, G. et al. Corrected feed restriction prevents ascites. Poultry, v.6, n.2, p.22-23, 1990.

ALBERS, G.A.A. Future trends in poultry breeding. In: EUROPEAN POULTRY CONFERENCE, 10., 1998, Jerusalem, Israel. Proceedings... Jerusalem : WPSA, 1998. p.16-20.

AVEWORLD. Entrevista: Zoe Silveira d'Ávila. Aveworld, v.1, n.1, p.4-8, 203.

BEKER, A. et al. Graded atmospheric oxygen level effects on performance and ascites incidence in broilers. Poultry Science, v.82, n.10, p.1550-1553, 2003.
BOTTJE, W.G.; HARRISON, P.C. The effect of tap water, carbonated water, sodium bicarbonate, and calcium chloride on blood acid-base balance in cockerels subjected to heat stress. Poultry Science, v.64, n.1, p.107-113, 1985.

BRANTON, S.L.; REECE, F.N.; DEATON, J.W. Use of ammonium chloride and sodium bicarbonate in acute heat exposure of broilers. Poultry Science, v.65, n.9, p.1659-1663, 1986.

BUYS, N. et al. Intermitent lighting reduces the incidence of ascites in broilers: An interaction with protein content of feed on performance and the endocrine system. Poultry Science, v.77, n.1, p.54-61, 1998.

CUEVA, S. et al. High altitude induced pulmonary hypertension and right heart failure in broiler chickens. Research in Veterinary Science, v.16, n.3 p.370-374, 1974.

DALE, N. Dietary factors influence ascites syndrome in broilers. Feedstuffs, v.24, p.14-16, 1990.

DECUYPERE, E., BUYSE, J., BUYS, N. Ascites in broiler chickens: exogenous and endogenous structural and functional causal factors. World's Poultry Science Journal, v.56, n.4, p.367-377, 2000.

FEDDE, M.R.; WEIGLE G.E.; WIDEMAN Jr., R.F. Influence of feed deprivation on ventilation and gas exchange in broilers: Relationship to pulmonary hypertension syndrome. Poultry Science, v.77, n.11, p.1704-1710, 1998.

FEDDE, M.R.; WIDEMAN, R.F. Blood viscosity in broilers: influence on pulmonary hypertension syndrome. Poultry Science, v.75, n.10, p.1261-1267, 1996.

FURLAN, R.L. et al. Alterações hematológicas e gasométricas em diferentes linhagens de frangos de corte submetidos ao estresse calórico agudo. Revista Brasileira de Ciência Avícola, v.1, n.1, p.77-84, 1999.

GONZALES, E. et al. Metabolic disturbances in male broilers of different strains. 2. Relationship between the thyroid and somatotrophic axes with growth rate and mortality. Poultry Science, v.78, n.2, p.516-521, 1999.

GONZALES, E.; MACARI, M. Enfermidades metabólicas em frangos de corte. In: BERCHIERI Jr., A.; MACARI, M. Doenças das aves. Campinas : FACTA, 2000. p.451-464.

HERNANDES, R. Efeito da exposição ao frio sobre a resposta pulmonar e cardíaca de $\mathbf{H s p} \mathbf{7 0}$ em frangos de corte resistentes e suscetíveis à síndrome ascítica. 2000. 100f. Dissertação (Mestrado em Zootecnia) - Faculdade de Ciências Agrárias e Veterinárias, Universidade Estadual Paulista.

HERNANDEZ, A. Hypoxic ascites in broilers: a review of several studies done in Colombia. Avian Diseases, v.31, n.3, p.658-661, 1987.

HUCHZERMEYER, F.W.; DERUYCK, A.M.C. Pulmonary hypertension syndrome associated with ascites in broilers. Veterinary Record, v.199, n.4, p.94, 1986.

JAENISCH, F.R. et al. Utilização de pinteiros cobertos (estufas) na redução da síndrome ascítica em frangos de corte. In: CONFERÊNCIA APINCO DE CIÊNCIA E TECNOLOGIA AVÍCOLAS, 1995, Curitiba, Brasil. Trabalhos de Pesquisa... Campinas : FACTA, 1995. p.157-158. 
JULIAN, R.J. Ascites and edema. In: London : Bailliere Tindall, 1990. p.343-353.

Poultry diseases.

JULIAN, R.J. Ascites in poultry. Avian Pathology, v.22, n.3 p.419-454, 1993.

JULIAN, R.J. Cardiovascular diseases. In: JORDAN, F.T.W.; PARRISON, M. Poultry diseases. London : Saunders, 1996. p.343-374.

JULIAN, R.J. The effect of increased sodium in the drinking water on right ventricular hypertrophy, right ventricular failure and ascites in broiler chickens. Avian Pathology, v.16, n.1, p.6171, 1987.

JULIAN, R.J.; CASTON, L.J.; LEESON, S. The effect of dietary sodium on right ventricular failure induced ascites, gain and fat deposition in meat-type chickens. Canadian Journal of Veterinary Research, v.56, n.3, p.214-219, 1992.

JULIAN, R.J.; McMILLAN, I.; QUINTON, M. The effect of cold and dietary energy on right ventricular hypertrophy, right ventricular failure and ascites in meat-type chickens. Avian Pathology, v.18, n.4, p.675-684, 1989.

JULIAN, R.J.; WILSON, B. Pen oxygen concentration and pulmonary hypertension-induced right ventricular failure and ascites in meat-type chickens at low altitude. Avian Diseases, v.36, n.3, p.733-735, 1992.

KANNAN, N.; SINGH, B. Changes in hematological and bloodgas variable in broilers at high-altitude with ascites syndrome. Indian Veterinary Journal, v.71, n.9, p.881-884, 1994.

LOTT, B.D.; BRANTON, S.L.; MAY, J.D. The effect of photoperiod and nutrition on ascites incidence in broilers. Avian Diseases, v.40, n.4, p.788-791, 1996.

LUBRITZ, D.L.; McPHERSON, B.N. Effect of genotype and cold stress on incidence of ascites in cockerels. Journal of Applied Poultry Research, v.3, n.2, p.171-178, 1994.

LUBRITZ, D.L.; SMITH, J.L.; McPHERSON, B.N. Heritability of ascites and the ratio of right to total ventricle weight in broiler breeder male lines. Poultry Science, v.74, n.7, p.1237-1241, 1995.

MACARI, M.; FURLAN, R.L.; GONZALES, E. Fisiologia cardiovascular. In: Fisiologia aviária aplicada a

frangos de corte. Jaboticabal : FUNEP/UNESP, 1994. p.12-25.

MAXWELL, M.H. et al. Comparison of hematological values in restricted and ad libitum fed domestic fowls: red blood cell characteristics. British Poultry Science, v.31, n.2, p.407-413, 1990.

MAXWELL, M.H.; ROBERTSON, G.W. Vision panorámica de la ascitis en pollos en el mundo: 1996. Revista Industria Avícola, Edicion Latinoamericana de Poultry Internacional, p.14-25, mai, 1997.

MAXWELL, M.H.; ROBERTSON, G.W.; McCORQUODALE, C.C. Whole blood and plasma viscosity values in normal and ascitic broiler chickens. British Poultry Science, v.33, n.4, p.871-877, 1992.

MAXWELL, M.H.; ROBERTSON, G.W.; SPENCE, S. Studies on an ascitic syndrome in young broilers. 1. Hematology and pathology. Avian Pathology, v.15, n.3, p.511-525, 1986.
McGOVERN, R.H. et al. Analysis of right ventricular areas to assess the severity of ascites syndrome in broiler chickens. Poultry Science, v.78, n.1, p.62-65, 1999a.

McGOVERN, R.H. et al. Growth performance, carcass characteristics, and the incidence of ascites in broilers in response to feed restriction and litter oiling. Poultry Science, v.78, n.4, p.522-528, 1999b.

McGOVERN, R.H. et al. Growth, carcass characteristics, and incidence of ascites in broilers exposed to environmental fluctuations and oiled litter. Poultry Science, v.79, n.3, p.324330, 2000

MIRSALIMI, S.M.; JULIAN, R.J. Reduced erythrocyte deformability as a possible contributing factor to pulmonary hypertension and ascites in broiler chickens. Avian Diseases, v.35, n.2, p.374-379, 1991.

OLKOWSKI, A.A. et al. Ultrastructural and molecular changes in the left and right ventricular myocardium associated with ascites syndrome in broiler chickens raised at low altitude. Journal of Veterinary Medicine Series A - Physiology, Pathology Clinical Medicine, v.48, n.4, p.1-14, 2001

OWEN, R.L. et al. Effect of age of exposure and dietary acidification or alkalization on broiler pulmonary hypertension syndrome. Journal of Applied Poultry Research, v.3, n.3, p.244-252, 1994

OWEN, R.L. et al. Physiological and electrocardiographic changes occurring in broilers reared at simulated high-altitude. Avian Diseases, v.39, n.1, p.108-115, 1995.

OWEN, R.L. et al. Use of a hypobaric chamber as a model system for investigating ascites in broilers. Avian Diseases, v.34, n.3, p.754-758, 1990

PEACOCK, A.J. et al. The relationship between rapid growth and pulmonary hemodynamics in the fast-growing broiler chicken. American Review of Respiratory Disease, v.139, n.6, p.1524-1530, 1989

PRICE, S.E.H.; DUNNINGTON, E.A.; SIEGEL, P.B. Hematocrit values in weight-selected and relaxed lines of white rock chickens. Poultry Science, v.77, n.10, p.1478-1480, 1998.

REEVES, J.T. et al. Improved arterial oxygenation with feed restriction in rapidly growing broiler chickens. Comparative Biochemistry and Physiology A-Physiology, v.99, n.3, p.481-485, 1991

REFFAY, M. Situation des productions avicoles. Nouzilly, France : INRA, Station de Recherches Avicoles, 1998. 126p.

ROSÁRIO, M.F. et al. Influência do genótipo e do sexo sobre o valor hematócrito em galinhas reprodutoras pesadas. Revista Brasileira de Ciência Avícola, v.2, n.3, p.281-286, 2000.

ROSÁRIO, M.F. et al. Uso da técnica do micro-hematócrito para predição do peso corporal e de características reprodutivas em frangos de corte. Revista Brasileira de Zootecnia, v.31, n.3, p.1396-1402, 2002 (Suplemento).

SANCHEZ, A. et al. Croissance musculaire et fonction cardiorespiratoire chez le poulet de chair. INRA Productions Animales, v.13, n.1, p.37-45, 2000. 
SAUVEUR, B. Stratégies pour de nouveaux progrès techniques et économiques en aviculture. INRA Productions Animales, v.4, n.1, p.31-40, 1991.

SCHEELE, C.W. et al. Ascites in broilers. 2.Disturbances in the hormonal regulation of metabolic rate and fat metabolism. Poultry Science, v.71, n.12, p.1971-1984, 1992.

SCHEELE, C.W. et al. Haematological characteristics predicting susceptibility for ascites. 1. High carbon dioxide tensions in juvenile chickens. British Poultry Science, v.44, n.3, p.476-483, 2003a.

SCHEELE, C.W. et al. Haematological characteristics predicting susceptibility for ascites. 2. High haematocrit values in juvenile chickens. British Poultry Science, v.44, n.3, p.484-489, 2003b.

SCHEELE, C.W.; FRANKENHUIS, M.T. Stimulation of the metabolic rate in broilers and the occurence of metabolic disorders. In: SYMPOSIUM ON ENERGY METABOLISM, 11., 1988, Lunteren, Netherlands. Proceedings... Wageningen : EAAP, 1988. p.251-254, n.43.

SCHEELE, C.W. Effects of nutritional factors on the occurrence of ascites and heart failure syndrome. In: EUROPEAN SYMPOSIUM ON POULTRY NUTRITION, 9., 1993, Jelenia Góra, Poland. Proceedings... Wroclaw : WPSA/Palma, 1993. p.149-162.

SHLOSBERG, A. et al. Controlled early feed restriction as a potencial means of reducing the incidence of ascites in broilers. Avian Diseases, v.35, n.4, p.681-684, 1991.

SHLOSBERG, A. et al. Relationship between broiler chicken hematocrit-selected parents and their progeny, with regard to hematocrit, mortality from ascites and body weight. Research in Veterinary Science, v.64, n.2, p.105-109, 1998.

SHLOSBERG, A. et al. The effects of poor ventilation, low tempertaures, type of feed and sex of bird on the development of ascites in broilers. Physiological factors. Avian Pathology, v.21, n.3, p.369-382, 1992

SILVERSIDES, F.G.; LEFRANÇOIS, M.F.; VILLENEUVE, P. The effect of strain of broiler on physiological parameters associated with the ascites syndrome. Poultry Science, v.76, n.5, p.663-667, 1997.

STURKIE, P.D.; GRIMINGER, P. Blood: physical characteristics, formed elements, hemoglobin, and coagulation. In: STURKIE, P.D. Avian physiology. New York : SpringerVerlag, 1976. p.53-76.

TANKSON, J.D.; THAXTON, J.P.; VIZZIER-THAXTON, Y. Pulmonary hypertension syndrome in young chickens challenged with frozen and autoclaved cultures of Enterococcus falecalis. Experimental Biology and Medicine, v.227, n.9, p.812-816, 2002a.

TANKSON, J.D.; THAXTON, J.P.; VIZZIER-THAXTON, Y. Biochemical and immunological changes in chickens experiencing pulmonary hypertension syndrome caused by Enterococcus faecalis. Poultry Science, v.81, n.12, p.1826-1831, 2002 b.

WIDEMAN, R.F. Ascites in poultry. Monsanto Nutricional, Update v.6, p.1-7, 1988.

WIDEMAN, R.F.,et al. Evaluation of minimally invasive indices for predicting ascites susceptibility in three successive hatches of broilers exposed to cool temperatures. Poultry Science, v.77, n.10, p.1565-1573, 1998.

WILLIAMS, R.B. The ratio of the water and food consumption of chickens and its significance in the chemotherapy of coccidiosis. Veterinary Research Communications, v.20, n.5, p.437-447, 1996.

YERSIN, A.G. et al. Changes in hematological, blood-gas, and serum biochemical variables in broilers during exposure to simulated high-altitude. Avian Diseases, v.36, n.2, p.189-196, 1992. 